\title{
Alkaline phosphatase variation during carfilzomib treatment is associated with best response in multiple myeloma patients
}

Maurizio Zangari ${ }^{1}$, Monette Aujay ${ }^{2}$, Fenghuang Zhan ${ }^{1}$, Kristina L. Hetherington ${ }^{1}$, Tamara Berno ${ }^{3}$, Ravi $\mathrm{Vij}^{4}$, Sundar Jagannath ${ }^{5}$, David Siegel ${ }^{6}, \mathrm{~A}$. Keith Stewart ${ }^{7}$, Luhua Wang ${ }^{8}$, Robert Z. Orlowski ${ }^{8}$, Andrew Belch $^{9}$, Andrzej Jakubowiak ${ }^{10}$, George Somlo $^{11}$, Suzanne Trudel ${ }^{12}$, Nizar Bahlis ${ }^{13}$, Sagar Lonial ${ }^{14}$, Seema Singhal ${ }^{15}$, Vishal Kukreti ${ }^{12}$, Guido Tricot $^{1}$

${ }^{1}$ Division of Hematology, University of Utah, Blood/Marrow Transplant and Myeloma Program, Salt Lake City, UT, USA; ${ }^{2}$ Department of Cancer Biology, Stem Cent Rx, LLC, South San Francisco, CA, USA; ${ }^{3}$ Department of Hematology and Clinical Immunology, University of Padua, Padua, Italy; ${ }^{4}$ BMT and Leukemia Program, Washington University, St Louis, MO, USA; ${ }^{5}$ Department of Hematology, Mount Sinai Medical Center, New York, NY, USA; ${ }^{6}$ Myeloma Division, John Theuer Cancer Center, Hackensack, NJ, USA; ${ }^{7}$ Mayo Clinic, Phoenix/Scottsdale, Scottsdale, AZ, USA; ${ }^{8} \mathrm{MD}$ Anderson Cancer Center, Houston, TX, USA; ${ }^{9}$ University of Alberta, Edmonton, Canada; ${ }^{10}$ Myeloma Program, University of Michigan Cancer Center, Ann Arbor, MI, USA; ${ }^{11}$ City of Hope National Medical Center, Duarte, CA, USA; ${ }^{12}$ Princess Margaret Hospital, Toronto, Canada; ${ }^{13}$ Division of Hematology, University of Calgary, Calgary, Canada; ${ }^{14}$ Winship Cancer Institute of Emory University, Atlanta, GA, USA; ${ }^{15}$ Multiple Myeloma Program, Northwestern University Medical School/Robert H. Lurie Comprehensive Cancer Center, Chicago, IL, USA

\begin{abstract}
The ubiquitin-proteasome pathway regulates bone formation through osteoblast differentiation. We analyzed variation alkaline phosphatase (ALP) during carfilzomib treatment. Data from 38 patients enrolled in the PX-171-003 and 29 patients in PX-171-004 studies, for patients with relapsed/refractory myeloma, were analyzed. All patients received $20 \mathrm{mg} / \mathrm{m}^{2}$ of carfilzomib on Days 1, 2, 8, 9, 15, and 16 of a 28-day cycle. Sixty-seven patients from ALP data were evaluable. In PX-171-003, the ORR (>PR) was 18\% and the clinical benefit response (CBR; $>M R$ ) was $26 \%$, while in PX-171-004, the ORR was $35.5 \%$ overall and $57 \%$ in bortezomib-naive patients. ALP increment from baseline was statistically different in patients who achieved $\geq$ VGPR compared with all others on Days $1(P=0.0049)$ and $8(P=0.006)$ of Cycle 2 . In patients achieving a VGPR or better, ALP increased more than 15 units per liter at Cycle 2 Day 1 over baseline. An ALP increase over the same period of time was seen in $26 \%, 13 \%$ and $11 \%$ of patients achieving PR, $M R$, and $S D$, respectively. This retrospective analysis of patients with relapsed or refractory myeloma treated with single-agent carfilzomib indicates that early elevation in ALP is associated with subsequent myeloma response.
\end{abstract}

Key words multiple myeloma; alkaline phosphatase; carfilzomib

Correspondence Maurizio Zangari, MD, School of Medicine, University of Utah, 30 N. 1900 E., Room 5C402, Salt Lake City, UT 84132, USA. Tel: 801585 3229; Fax: 801585 3432; e-mail: maurizio.zangari@hsc.utah.edu

Accepted for publication 3 March 2011

doi:10.1111/j.1600-0609.2011.01602.x

Multiple myeloma (MM) is a plasma cell malignancy with impaired bone formation. In myeloma, RANKL expression is markedly increased while its decoy receptor, osteoprotegerin (OPG), is decreased (1). The Wnt signaling inhibitor DKK1 expression is significantly increased and correlates with the extent of bone disease $(2,3)$. The ubiquitin-proteasome pathway, an essential cellular deg- radative system in myeloma cells, can also regulate bone formation via effects on osteoblast differentiation $(4,5)$. Bortezomib, the first proteasome inhibitor, has been shown to have inhibitory effect on myeloma SCID-hu model in vivo. Primary human myeloma cells are grown in a human fetal bone, engrafted in the flank of SCID mice. In this model, the use of bortezomib $(0.5 \mathrm{mg} / \mathrm{kg}$ 
twice a week) was associated with disease control and increase in bone mineral density (BMD) $(20 \pm 14 \%)$, while myeloma growth induced a decrease in BMD $(13 \pm 12 \%)$ and progressive tumor in untreated animals. Exposure of non-myeloma-bearing control mice to bortezomib also resulted in a significant increase in BMD (6). While osteoprogenitor differentiation is not affected by immunomodulatory analogues (lenalidomide, CC-4047, CC-6032), both bortezomib and immunomodulatory compounds retain inhibitory effect on osteoclast differentiation $(7,8)$. Retrospective analysis of the bone alkaline phosphatase (ALP) variation during treatment of myeloma patients with bortezomib has indicated a close correlation between myeloma response and drug activity (9). An analysis of the APEX data showed that a $25 \%$ increase in ALP after 6 wks' treatment was the best predictor of myeloma response and was associated with time to progression (10).

The phase 3 Vista trial compared the use of melphalan and prednisone with or without bortezomib in previously untreated patients with MM who were ineligible for a high-dose therapy. Six hundred and eighty-two patients were randomly assigned to receive nine 6-week cycles of melphalan and prednisone either alone or with bortezomib (11). A retrospective analysis showed that rates of bisphosphonates use and bone disease events were significantly lower in patients enrolled in the VMP arm compared with the MP control arm (12).

Carfilzomib is a novel inhibitor that binds selectively and irreversibly to the proteasome resulting in greater and more sustained inhibition compared with bortezomib, and in vitro studies have shown to be able to overcome bortezomib resistance (13). Carfilzomib selectively inhibits the N-terminal threonine protease activity of the proteasome with minimal cross-reactivity with serine and other proteases. This analysis is the first to focus on the variations of ALP and myeloma response in carfilzomibtreated patients with myeloma.

\section{Material and methods}

Retrospective analysis of the relationship between serum ALP and tumor response was performed on patients with relapsed or refractory myeloma.

Analysis of serum ALP variation was completed in patients with myeloma enrolled on two phase 2 studies (PX-171-003 and PX-171-004) evaluating the safety and efficacy of single-agent carfilzomib dosed at $20 \mathrm{mg} / \mathrm{m}^{2}$. We analyzed data from 38 patients in the PX-171-003-A0 study, a relapsed and refractory myeloma trial for patients who have received $\geq 3$ prior therapies including bortezomib and an immunomodulatory drug (IMiD), along with 29 patients in PX-171-004, a relapsed or refractory myeloma trial that included bortezomib-naive patients. All patients received $20 \mathrm{mg} / \mathrm{m}^{2}$ of carfilzomib on Days 1, 2, 8, 9, 15, and 16 of a 28-day cycle and remained on study until progression, intolerable adverse events, or withdrawal of consents.

Serum ALP measurements were taken prior to the first dose of carfilzomib (baseline) and periodically during the trial. Change in ALP levels from baseline to Cycle 2 Day 1 was measured in a group of 117 patients but only 67 patients with ALP data were evaluable for response. ALP data were aligned with clinical response data and (as per international myeloma working group (IMWG) response criteria) with ALP variations associated with response groups. All patients enrolled in this study have signed informed consent approved by the IRB.

\section{Results}

Sixty-seven patients were included in this analysis. The median age was 63 yrs with a median time since diagnosis of $4.6 \mathrm{yrs}, 52 \%$ were men, $84 \%$ had relapsed after Autologous stem cell transplantations, 82\% were previously exposed to bortezomib, and $92 \%$ were previously exposed to an IMiD (Table 1). In PX-171-003, the overall response rate (ORR) ( $\geq \mathrm{PR}$ ) was $18 \%$ and the clinical

Table 1 Patients characteristics

\begin{tabular}{|c|c|c|c|c|}
\hline \multirow[b]{4}{*}{ Characteristic } & \multicolumn{4}{|c|}{ Number of $(\%)$ of subjects } \\
\hline & \multirow[b]{2}{*}{ 003-A0 } & \multicolumn{2}{|l|}{004} & \multirow[b]{2}{*}{ Total } \\
\hline & & Bortezomib treated & Bortezomib naive & \\
\hline & $(N=38)$ & $(N=29)$ & $(N=51)$ & $(N=117)$ \\
\hline Median age (years) & 64 & 63 & 64 & 64 \\
\hline Median years since diagnosis & 5.9 & 3.6 & 3.5 & 4.3 \\
\hline Gender: male & $19(51.4)$ & $14(48.3)$ & $28(54.9)$ & $61(52.1)$ \\
\hline Mean number of prior therapies & 6.1 & 2.9 & 2.0 & 3.5 \\
\hline Received bortezomib & $37(100.0)$ & $29(100.0)$ & $0(0.0)$ & $66(54.5)$ \\
\hline Received IMiD & $37(100.0)$ & $22(75.9)$ & $47(92.2)$ & $106(90.6)$ \\
\hline Received transplant & $30(81.1)$ & $25(86.2)$ & $41(80.4)$ & $96(82.1)$ \\
\hline Refractory to most recent therapy & $37(100)$ & $9(31)$ & $20(39)$ & $66(56)$ \\
\hline
\end{tabular}



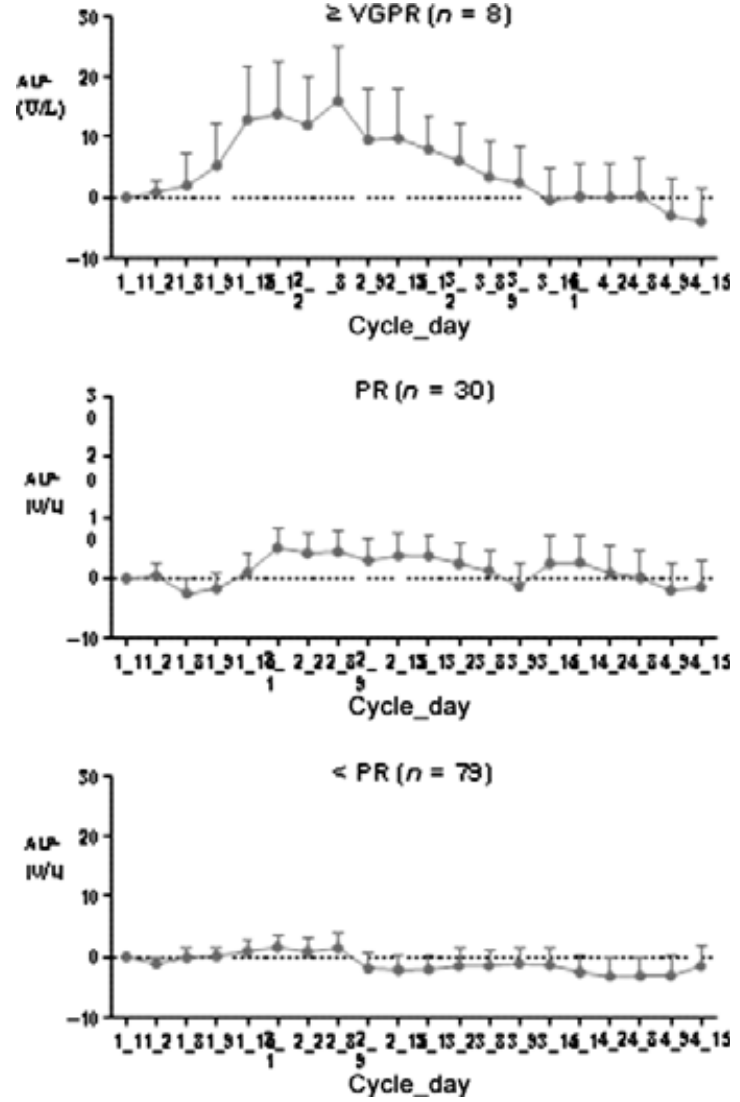

Figure 1 Average alkaline phosphatase change from baseline increase associated with response.

benefit response $(\mathrm{CBR} ; \geq \mathrm{MR})$ was $26 \%$, while in PX171-004, the ORR was $35.5 \%$ overall and $57 \%$ in bortezomib-naive patients. Average ALP change from baseline appeared to be associated with responses. Figure 1 shows ALP variation during treatment in patients according to clinical response.

ALP increment from baseline, which was most evident during the second cycle of treatment, was statistically different in patients who achieved $\geq$ very good partial remission (VGPR) compared with all others on Days 1 $(P=0.0049)$ and $8(P=0.006)$ of Cycle 2 . In all patients achieving a VGPR or better, ALP increased more than 15 units per liter at Cycle 2 Day 1 over baseline. An ALP increase over the same period of time was seen in $26 \%, 13 \%$ and $11 \%$ of patients achieving PR, $\mathrm{MR}$, and SD, respectively.

None of the patients with progressive disease exhibited a similar increase. Our study indicates that response first assessed on Day 15 of Cycle 1 parallels the ALP elevation that returned to baseline levels at the end of Cycle 3 . Twenty-nine percent $(11 / 38)$ of subjects achieving at least a PR had an increase in ALP of $>15 \mathrm{U} / \mathrm{L}$ from baseline, while only $7.6 \%(6 / 79)$ of subjects failing to achieve at least a PR showed a similar increase. Of the 17 patients that had increases of $>15 \mathrm{U} / \mathrm{L}, 11$ patients $(65 \%)$ responded ( $>$ PR) to carfilzomib treatment. An increase in serum ALP levels on Cycle 2 Day 1 from baseline is associated with best response.

\section{Discussion}

Several in vivo studies have shown that proteasome inhibition is associated with an increase in bone formation. Pennisi et al. (6) reported a significant $(P<0.04)$ increase in BMD in SCID-rab mice engrafted with MM cells treated with bortezomib; increases in bone volume, trabecular thickness, and bone formation were also observed in bortezomib-treated mice, both in myelomatous and non-myelomatous bones. Similarly, a decrease in osteolytic lesions and increases in trabecular number and bone volume have been reported following bortezomib treatment of 5T2MM mice (14).

In a murine model of MM, bortezomib-treated mice showed a $400 \%$ increase in osteoblast numbers compared with vehicle mice. Furthermore, the bortezomib-treated mice had around $50 \%$ more osteoblasts than tumornaive mice, suggesting a more direct effect of bortezomib on osteoblasts than was seen on osteoclasts in the same study (14).

This is supported by a previous in vitro study that demonstrated a direct effect of bortezomib in enhancing differentiation of fetal human MSCs into osteoblasts (6).

A study by De Matteo et al. (15) showed that osterix, a key transcription factor required for osteoblast differentiation, is down-regulated in MM but can be up-regulated by bortezomib.

In a prospective phase 2 study, bortezomib was associated with a statistically significant increase from baseline in bone volume/total volume (as assessed via comparative histomorphometric microCT analysis) in six of seven patients with relapsed/refractory MM. This study has also indicated that response to bortezomib was associated with changes in serum parathyroid hormone (PTH) concentrations (16).

This retrospective analysis on a subset of patients in these ongoing phase 2 studies of single-agent carfilzomib in relapsed or refractory MM has shown for the first time that elevation in serum ALP during carfilzomib is associated with best tumor responses. Sixty-five percent $(11 / 17)$ of subjects with an increase in ALP of $>15 \mathrm{U} / \mathrm{L}$ above baseline achieved a PR or better. Initial tumor response paralleled the ALP elevation, with ALP values generally returning to baseline levels at the end of Cycle 3 even if the response seems to continue. PTH levels were not measured in these phase 2 studies of carfilzomib.

Taken with previous publications describing bortezomib treatment, these results suggest that this specific ana- 
bolic bone phenomenon could be a class effect of proteasome inhibitors. Additional phase 2 studies with a higher dose of carfilzomib (i.e. $27 \mathrm{mg} / \mathrm{m}^{2}$ ) are ongoing. The data from this small subset analysis suggest that further exploration of this relationship between bone-derived markers and myeloma response is warranted.

\section{Author contributions}

Conception and design: Maurizio Zangari, Guido Tricot, Monette Aujay; Provision of study materials or patients: Ravi Vij, Sundar Jagannath, David Siegel, A. Keith Stewart, Luhua Wang, Robert Z. Orlowski, Andrew Belch, Andrzej Jakubowiak, George Somlo, Suzanne Trudel, Nizar Bahlis, Sagar Lonial, Seema Singhal, Vishal Kukreti; Collection and assembly of data: Monette Aujay, Tamara Berno; Data analysis and interpretation: Maurizio Zangari, Monette Aujay; Manuscript writing: Maurizio Zangari, Guido Tricot, Kristina L. Hetherington; Final approval of manuscript: Maurizio Zangari, Guido Tricot.

\section{References}

1. Pearse RN, Sordillo EM, Yaccoby S, Wong BR, Liau DF, Colman N, Michaeli J, Epstein J, Choi Y. Multiple myeloma disrupts the TRANCE/osteoprotegerin cytokine axis to trigger bone destruction and promote tumor progression. Proc Natl Acad Sci USA 2001;98:11581-6.

2. Giuliani N, Rizzoli V, Roodman GD. Multiple myeloma bone disease: pathophysiology of osteoblast inhibition. Blood 2006;108:3992-6.

3. Tian E, Zhan F, Walker R, Rasmussen E, Ma Y, Barlogie $\mathrm{B}$, Shaughnessy JD Jr. The role of the Wnt-signaling antagonist DKK1 in the development of osteolytic lesions in multiple myeloma. $N$ Engl J Med 2003;349:2483-94.

4. Garrett IR, Chen D, Gutierrez G, et al. Selective inhibitors of the osteoblast proteasome stimulate bone formation in vivo and in vitro. $J$ Clin Invest 2003;111:1771-82.

5. Mukherjee S, Raje N, Schoonmaker JA, et al. Pharmacologic targeting of a stem/progenitor population in vivo is associated with enhanced bone regeneration in mice. $J$ Clin Invest 2008;118:491-504.

6. Pennisi A, Li X, Ling W, Khan S, Zangari M, Yaccoby S. The proteasome inhibitor, bortezomib suppresses primary myeloma and stimulates bone formation in myelomatous and nonmyelomatous bones in vivo. Am J Hematol 2009;84:6-14.

7. Munemasa S, Sakai A, Kuroda Y, Okikawa Y, Katayama Y, Asaoku H, Kubo T, Shimose S, Kimura A. Osteoprogenitor differentiation is not affected by immunomodulatory thalidomide analogs but is promoted by low bortezomib concentration, while both agents suppress osteoclast differentiation. Int J Oncol 2008;33:129-36.

8. Breitkreutz I, Raab MS, Vallet S, et al. Lenalidomide inhibits osteoclastogenesis, survival factors and boneremodeling markers in multiple myeloma. Lenalidomide, bortezomib inhibit osteoclasts in MM. Leukemia 2008;22:1925-32.

9. Zangari M, Esseltine D, Lee CK, et al. Response to bortezomib in associated to osteoblastic activation in patients with multiple myeloma. Br J Haematol 2005;131:71-3.

10. Zangari M, Esseltine D, Cavallo F, Neuwirth R, Elice F, Burns MJ, Yaccoby S, Richardson P, Sonneveld P, tricot G. Predictive value of alkaline phosphatase for response and time to progression in bortezomib-treated multiple myeloma patients. Am J Hematol 2007;82:831-3.

11. San Miguel JF, Schlag R, Khuageva NK, et al. Bortezomib plus melphalan and prednisone for initial treatment of multiple myeloma. N Engl J Med 2008;359:906-17.

12. Delforge M, Kropff M, Spicka I, et al. VMP results in fewer bone events and greater ALP increases versus MP in the VISTA study in front-line MM. Clin Lymphoma Myeloma 2009;9:S43 (abstr A246).

13. Suzuki E, Demo S, Arastu-Kapur S, Kirk CJ, Bennett MK. Bortezomib-resistant cell lines have increased proteasome levels but remain sensitive to carfilzomib. Blood 2009;114:2852 (abstract).

14. Deleu S, Lemaire M, Arts J, et al. Bortezomib alone or in combination with the histone deacetylase inhibitor JNJ26481585: effect on myeloma bone disease in the 5T2MM murine model of myeloma. Cancer Res 2009;69:5307-11.

15. De Matteo M, Brunetti AE, Maiorano E, Cafforio P, Dammacco F, Silvestris F. Constitutive down-regulation of Osterix in osteoblasts from myeloma patients: in vitro effect of Bortezomib and Lenalidomide. Leuk Res 2010;34:243-9.

16. Zangari M, Yaccoby S, Pappas L, et al. A prospective evaluation of the biochemical, metabolic, hormonal and structural bone changes associated with bortezomib response in multiple myeloma patients. Haematologica 2011;96:333-6. 